\title{
Cobalt-protoporphyrin enhances heme oxygenase 1 expression and attenuates liver ischemia/reperfusion injury by inhibiting apoptosis
}

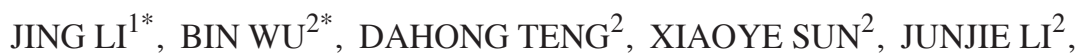 \\ JIANG $\mathrm{LI}^{2}$, GUOLIANG ZHANG ${ }^{3}$ and JINZHEN CAI ${ }^{2}$ \\ ${ }^{1}$ Departments of Transplantation and Gastroenterology, Tianjin Medical University, Tianjin 300070; \\ Departments of ${ }^{2}$ Transplantation and ${ }^{3}$ Gastroenterology, Tianjin First Central Hospital \\ of Tianjin Medical University, Tianjin 300192, P.R. China
}

Received March 14, 2016; Accepted February 27, 2017

DOI: $10.3892 / \mathrm{mmr} .2018 .8384$

\begin{abstract}
The aim of the present study was to investigate the preconditioning effect and underlying mechanisms of cobalt-protoporphyrin (CoPP) in a mouse model of liver ischemia-reperfusion (I/R) injury. Mice were divided into five groups: Sham-operated (control), I/R, I/R + CoPP, I/R + CoPP and zinc-protoporphyrin (ZnPP) and I/R + ZnPP. Serum levels of aspartate transaminase (AST) and alanine aminotransferase (ALT) were detected using commercial kits. The expression of the pro-apoptotic protein caspase- 3 was detected by immunohistochemistry and the expression levels of the anti-apoptotic protein B-cell lymphoma 2 (Bcl-2) and heme oxygenase 1 (HO-1) were analyzed by western blotting. Sections of liver tissue were stained with hematoxylin and eosin to observe pathologic alterations. Furthermore, hepatocyte apoptosis was detected using a terminal deoxynucleotidyl transferase dUTP nick end labeling (TUNEL) assay. AST and ALT levels of the CoPP preconditioned group were significantly reduced compared with the IR injury group $(\mathrm{P}<0.05)$ and liver damage was attenuated. The expression levels of the pro-apoptotic protein caspase 3 was inhibited and those of HO-1 and Bcl-2 were increased in the CoPP group compared with the I/R group; the opposite results were observed in the $\mathrm{ZnPP}$ group. Furthermore, the percentage of apoptotic cells as
\end{abstract}

Correspondence to: Dr Guoliang Zhang, Department of Gastroenterology, Tianjin First Central Hospital of Tianjin Medical University, 22 Fukang Road, Nankai, Tianjin 300192, P.R. China

E-mail: zgltianjin@sina.cn; zg1_022@163.com

Dr Jinzhen Cai, Department of Transplantation, Tianjin First Central Hospital of Tianjin Medical University, 22 Fukang Road, Nankai, Tianjin 300192, P.R. China

E-mail: caijinzhen@sina.com

${ }^{*}$ Contributed equally

Key words: cobalt-protoporphyrin, heme oxygenase-1, anti-apoptosis detected by TUNEL was significantly decreased in the CoPP group compared with the I/R group $(\mathrm{P}<0.05)$; these protective effects were abrogated by $\mathrm{ZnPP}$. In conclusion, the results of the present study suggested that CoPP may induce HO-1 overexpression and produce anti-apoptotic effects in liver I/R injury.

\section{Introduction}

With the increase of patients with end-stage liver disease requiring liver transplantation, the transplantation community has attempted to expand donor numbers using donation after cardiac death (DCD). Currently, DCD is a primary source of transplanted livers and a means of reducing the transplant waiting list. However, ischemia-reperfusion (I/R) injury is the most common cause of primary graft dysfunction (10-30\% of grafts) and primary graft nonfunction (5\% of grafts) for all liver transplants. Primary graft nonfuction results in $81 \%$ of retransplantation during the first week following surgery (1). Therefore, limiting the extent of I/R injury would improve the outcome for patients undergoing liver resection, particularly for recipients of DCD and fatty donor livers. However, the exact molecular mechanisms underlying I/R injury-associated innate immune cell activation remain to be fully elucidated.

Heme oxygenase $(\mathrm{HO})$ is an enzyme specialized in degrading heme and is assembled with biliverdin, carbon monoxide and free iron $(2,3)$. The upregulation of heme oxygenase 1 (HO-1) may provide protection from cellular stress following ischemia and inflammation, inhibiting the damage caused by heme and exerting anti-inflammatory and anti-apoptotic effects. HO-1 overexpression is cytoprotective in transplant models of hepatic I/R injury, with recipients exhibiting improved liver architecture and function, increased survival, and reduced inflammation (4). However, certain studies have suggested that these effects may be limited to a narrow window of HO-1 concentration $(5,6)$.

Cobalt-protoporphyrin (CoPP) is considered to be the most effective metalloporphyrin inducer of HO-1 $(1,7,8)$. The present study investigated the anti-apoptotic mechanisms underlying HO-1-induced cytoprotection using CoPP and the 
HO-1 inhibitor zinc-protoporphyrin (ZnPP) (9) in a mouse model of liver I/R injury.

\section{Materials and methods}

Animals and experimental model. Fifteen C57BL/6J mice (weight, 25-30 g) were purchased from the Laboratory Animal Center of the Academy of Military Medical Sciences (Beijing, China) and were housed in the animal facilities of Tianjin Medical University (Tianjin, China) at $23-25^{\circ} \mathrm{C}$ and $45-55 \%$ humidity with a $12 \mathrm{~h} \mathrm{light/dark} \mathrm{cycle.} \mathrm{Mice} \mathrm{had} \mathrm{free} \mathrm{access}$ to food and water. All mice were handled according to the Guidelines for the Care and Use of Laboratory Animals (National Institutes of Health, Bethesda, MD, USA) (10). Mice were denied solid food for $\sim 12 \mathrm{~h}$ and liquid food for $\sim 4 \mathrm{~h}$ prior to surgery. Liver I/R injury was induced in mice as previously described (11), following anesthesia with an intraperitoneal injection of $4 \%$ chloral hydrate $(10 \mathrm{mg} / 100 \mathrm{~g})$. Following a midline laparotomy, all structures in the portal triad (hepatic artery, portal vein and bile duct) were occluded with a vascular traumatic clamp. The blood supply to the liver was occluded for $60 \mathrm{~min}$ followed by $2 \mathrm{~h}$ reperfusion. Mice were scarified after reperfusion with an overdose of anesthetic. Mice were randomly assigned to five groups ( $\mathrm{n}=3 /$ group): i) Control (sham-operated); ii) I/R; iii) CoPP pretreatment, in which mice received an intraperitoneal injection of $5 \mathrm{mg} / \mathrm{kg}$ body weight CoPP (Sigma-Aldrich; Merck KGaA, Darmstadt, Germany) $48 \mathrm{~h}$ prior to I/R; iv) CoPP and ZnPP pretreatment, in which mice received an intraperitoneal injection of CoPP $48 \mathrm{~h}$ prior to I/R and an intraperitoneal injection of $15 \mathrm{mg} / \mathrm{kg}$ body weight ZnPP (Sigma-Aldrich; Merck KGaA) $24 \mathrm{~h}$ prior to $\mathrm{I} / \mathrm{R}$; and v) $\mathrm{ZnPP}$ pretreatment, in which mice received an intraperitoneal injection of $15 \mathrm{mg} / \mathrm{kg}$ body weight $\mathrm{ZnPP} 48 \mathrm{~h}$ prior to $\mathrm{I} / \mathrm{R}$.

Aspartate transaminase (AST) and alanine aminotransferase (ALT) detection. Serum was collected from all mice from the inferior vena cava at $2 \mathrm{~h}$ following reperfusion. The levels of AST and ALT in serum was determined using the following commercial kits: Aspartate Aminotransferase (AST) reagent OSR6509 and Alanine Aminotransferase (ALT) reagent OSR6607 (Beckman Coulter, Inc., Brea, CA, USA), according to the manufacturer's protocol.

Histological examination. Liver samples harvested from mice were fixed in formalin for $24 \mathrm{~h}$, embedded in paraffin and sectioned (4- $\mu \mathrm{m}$ thick). Sections were stained with hematoxylin and eosin (H\&E) to detect histological alterations. $\mathrm{H} \& \mathrm{E}$ staining was analyzed under a light microscope by two pathologists.

Western blotting. Liver tissues $(20 \mathrm{mg})$ were lysed with radioimmunoprecipitation assay buffer (Beijing SolarBio Science $\&$ Technology Co., Ltd., Beijing, China) and the proteins were extracted from the lysates following centrifugation at $13,363 \times \mathrm{g}, 4^{\circ} \mathrm{C}$ for $15 \mathrm{~min}$. Protein concentration was determined using a Bicinchoninic Acid Protein assay kit (Pierce; Thermo Fisher Scientific, Inc., Waltham, MA, USA). Liver proteins $(40 \mu \mathrm{g} /$ lane) were separated on $10 \%$ SDS-PAGE gels and transferred onto polyvinylidene difluoride membranes at
$250 \mathrm{~mA}$ for $2 \mathrm{~h}$. Non-specific proteins were blocked by incubating membranes in 5\% non-fat milk powder. Membranes were incubated with the following primary antibodies overnight at $4^{\circ} \mathrm{C}$ : Mouse monoclonal anti- $\beta$-actin $(1: 1,000$, cat no. $3700 \mathrm{~S})$, mouse $\mathrm{mAb}$ anti-B-cell lymphoma 2 [(Bcl-2); 1:200; cat no. 15071S] (both from Cell Signaling Technology, Inc., Danvers, MA, USA) and mouse monoclonal anti-HO-1 (1:500; ab13248; Abcam, Cambridge, MA, USA). Membranes were subsequently washed with TBS containing Tween-20 (TBST) three times for 10 min each time and incubated with anti-mouse IgG, horseradish peroxidase-conjugated secondary antibodies (1:500, cat no. 7076P2; Cell Signaling Technology, Inc.) for $2 \mathrm{~h}$ at room temperature. Following incubation, the membranes were washed with TBST three times for $10 \mathrm{~min}$ each time. Enhanced Chemiluminescence (EMD Millipore, Billerica, MA, USA) was performed according to the manufacturer's protocol. Western blots were quantified using Image Studio Digits 4.0 (LI-COR C-DiGit Blot Scanner; LI-COR Biosciences, Lincoln, NE, USA) and expression of proteins were normalized against $\beta$-actin expression.

Immunohistochemistry. Paraffin-embedded liver sections (4- $\mu \mathrm{m}$ thick) were dewaxed using xylene and hydrated through graded ethanol. Antigen retrieval was performed at $95^{\circ} \mathrm{C}$ for 15 min using citric acid buffer $(10 \mathrm{mM}$ citric acid, pH 6.0) in a microwave. Non-specific proteins were blocked using $3 \% \mathrm{H}_{2} \mathrm{O}_{2}$. Sections were subsequently incubated with a rabbit polyclonal anti-activated caspase- 3 antibody (1:100; cat no. 9662S; Cell Signaling Technology, Inc.) at $4^{\circ} \mathrm{C}$ overnight and incubated with a horseradish peroxidase-conjugated goat anti-rat polyclonal secondary antibody (dilution, 1:100; cat no. ab6721; Abcam) at $25^{\circ} \mathrm{C}$ for $1 \mathrm{~h}$. Color was developed using 3,3-diaminobenzidine (Sigma-Aldrich; Merck KGaA, Darmstadt, Germany) and sections were counterstained with hematoxylin. Sections were observed under a light microscope, and four fields per slide were randomly selected under $\mathrm{x} 400$ magnification for evaluation of caspase- 3 expression using Image-Pro Plus version 7.0 Image Analysis software (Media Cybernetics, Inc., Rockville, MD, USA).

Terminal deoxynucleotidyl transferase dUTP nick end labeling (TUNEL) assay. TUNEL assay (Roche Diagnostics, Indianapolis, IN, USA) was performed according to the manufacturer's protocol. Positive controls were obtained by incubating paraffin-embedded liver sections $(4-\mu \mathrm{m}$ thickness) with $1,500 \mathrm{U} / \mathrm{ml}$ DNase to induce DNA strand breaks prior to labeling. Sections incubated with labeling solution not containing terminal transferase served as a negative control (12). Apoptotic cells were imaged under a fluorescence microscope (Nikon Corporation, Tokyo, Japan). For each section, three non-overlapping fields of view (magnification, $\mathrm{x} 400$ ) were randomly selected. The percentage of apoptotic cells in each field was calculated as follows: (Apoptotic cell number/total cell number) x100 (13).

Statistical analysis. Data are expressed as the mean \pm standard deviation. SPSS software version 16.0 (SPSS, Inc., Chicago, IL, USA) was used to perform statistical analyses. Unpaired Student's t-test was used to compare differences between two groups, whereas one-way analysis of variance was performed 
Table I. Serum levels of liver enzyme AST and ALT following I/R injury.

\begin{tabular}{lcc}
\hline Group & AST (IU/1) & ALT (IU/l) \\
\hline Control & $39.11 \pm 14.73$ & $12.08 \pm 3.07$ \\
I/R & $114.93 \pm 19.22^{\mathrm{a}}$ & $29.94 \pm 5.21^{\mathrm{a}}$ \\
I/R with CoPP pretreatment & $45.35 \pm 9.35^{\mathrm{b}}$ & $16.75 \pm 4.80^{\mathrm{b}}$ \\
I/R with CoPP and ZnPP pretreatment & $117.35 \pm 16.02$ & $24.74 \pm 5.70$ \\
I/R with ZnPP pretreatment & $163.66 \pm 20.97^{\mathrm{b}}$ & $49.63 \pm 10.81^{\mathrm{b}}$
\end{tabular}

Data are expressed as the mean \pm standard deviation. ${ }^{a} \mathrm{P}<0.05 \mathrm{vs}$. control; ${ }^{\mathrm{b}} \mathrm{P}<0.05 \mathrm{vs}$. I/R. AST, aspartate transaminase; ALT, alanine aminotransferase; I/R, ischemia-reperfusion; CoPP, cobalt-protoporphyrin; ZnPP, zinc-protoporphyrin.
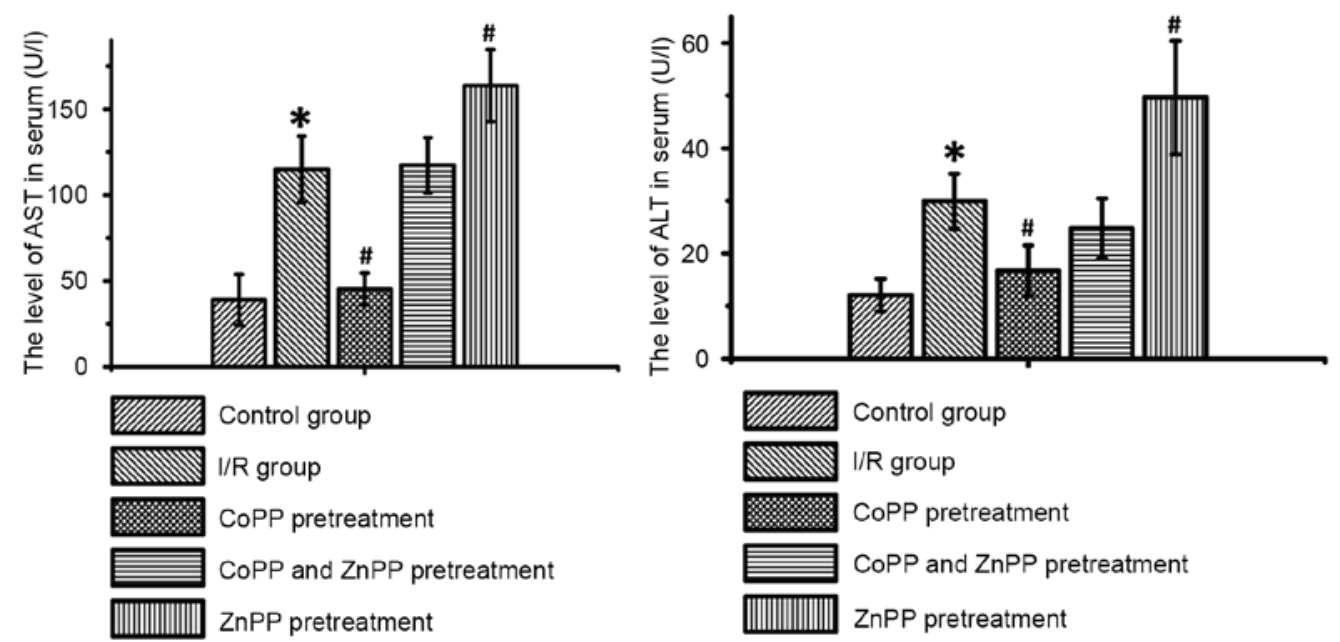

Figure 1. Serum levels of AST and ALT following I/R injury. The serum levels of AST and ALT were measured in mice following liver I/R injury, in the absence or presence of pretreatment with CoPP and/or ZnPP. Data are expressed as the mean \pm standard deviation. " $\mathrm{P}<0.05$ vs. control; ${ }^{*} \mathrm{P}<0.05$ vs. I/R. AST, aspartate transaminase; ALT, alanine aminotransferase; I/R, ischemia-reperfusion; CoPP, cobalt-protoporphyrin; ZnPP, zinc-protoporphyrin.

to compare multiple groups, followed by a Tukey's test. $\mathrm{P}<0.05$ was considered to indicate a statistically significant difference.

\section{Results}

CoPP pretreatment decreases serum levels of AST and ALT following I/R. As presented in Table I and Fig. 1, serum levels of liver enzymes in the sham-operated group remained in the normal range (ALT: 0-20 U/1, AST: 0-40 U/1). Following $\mathrm{I} / \mathrm{R}$, the levels of AST and ALT were significantly increased compared with the sham-operated group $(\mathrm{P}<0.05)$; CoPP pretreatment significantly inhibited this increase $(\mathrm{P}<0.05)$. ZnPP pretreatment increased the serum levels of AST and ALT compared with the I/R only group $(\mathrm{P}<0.05)$; however, there were no significant differences in AST and ALT levels between the CoPP and ZnPP pretreated group and the I/R only group.

CoPP pretreatment alleviates liver injury following $I / R$. To investigate histopathological alterations following I/R, $\mathrm{H} \& \mathrm{E}$ staining was performed. The liver cells in the control sham-operated group were arranged in plates surrounding the sinusoids (Fig. 2A). In the I/R group, hepatocyte swelling, cytoplasm rarefaction, spotty necrosis scattering in hepatic

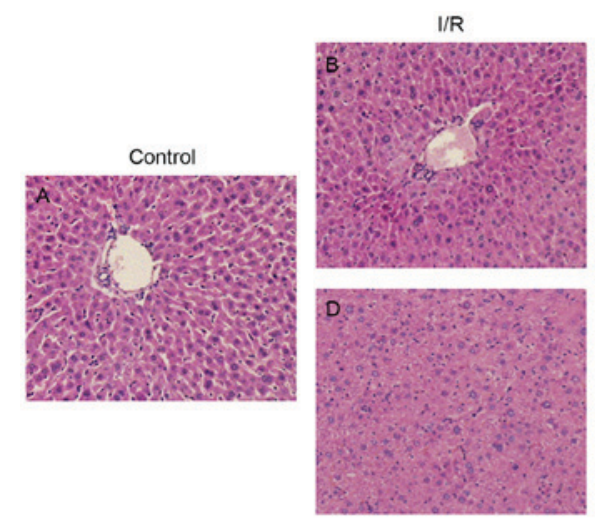

I/R with CoPP and ZnPP

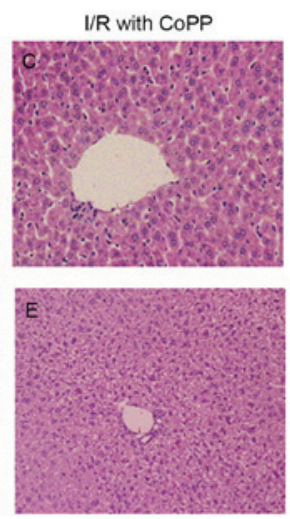

I/R with ZnPP
Figure 2. Hematoxylin and eosin staining of liver sections following I/R injury. Liver tissues were obtained from mice following liver I/R injury, in the absence or presence of pretreatment with CoPP and/or ZnPP. (A) Control (sham-operated) group, with liver cells are arranged in plates surrounding the sinusoids. (B) I/R group, with hepatocyte swelling, cytoplasm rarefaction, spotty necrosis scattering in hepatic lobules and inflammatory cell infiltration in portal duct areas. (C) I/R with CoPP pretreatment group, with cellular swelling alleviated and cellular morphology that appeared no different to the control group. (D) I/R with CoPP and ZnPP pretreatment group, with irregular arrangement and loss of portal duct. (E) I/R with ZnPP pretreatment group, with hydropic degeneration, irregular arrangement and narrow liver sinusoid. Original magnification, x100. I/R, ischemia-reperfusion; CoPP, cobalt-protoporphyrin; ZnPP, zinc-protoporphyrin. 

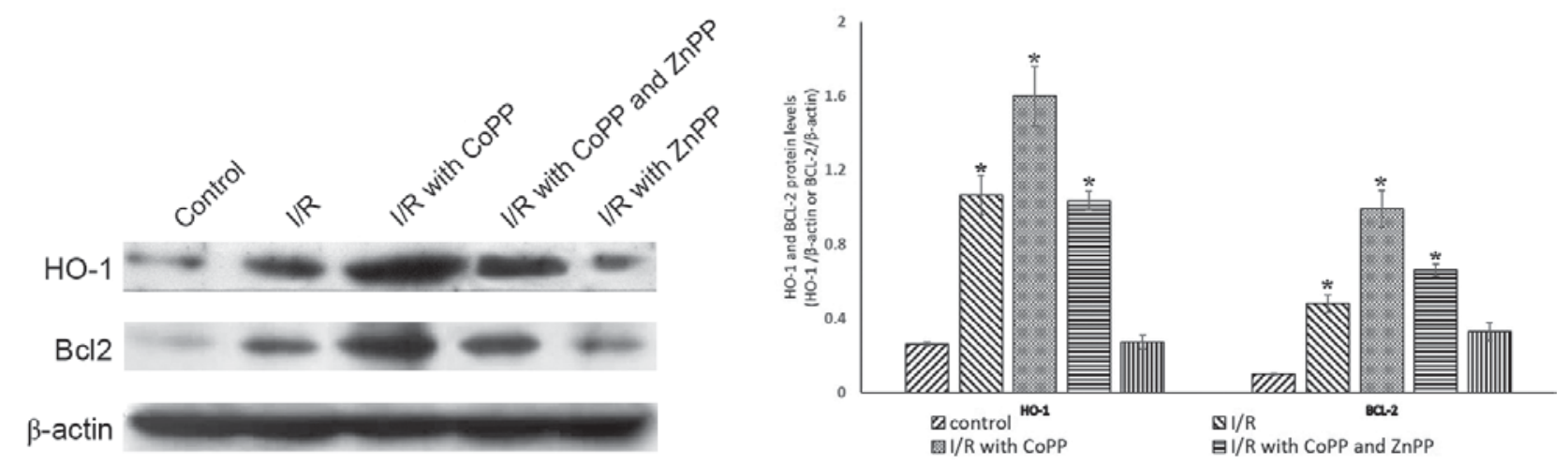

Figure 3. Protein expression levels of HO-1 and Bcl-2 following I/R injury. Liver tissues were obtained from mice following liver I/R injury, in the absence or presence of pretreatment with $\mathrm{CoPP}$ and/or ZnPP, and proteins were detected by western blotting. HO-1 protein expression levels were increased by CoPP and decreased by ZnPP pretreatment, and the expression level of Bcl-2 showed a similar pattern. * $\mathrm{P}<0.05$ vs. control. HO-1, heme oxygenase 1; Bcl-2, B-cell lymphoma 2; I/R, ischemia-reperfusion; CoPP, cobalt-protoporphyrin; ZnPP, zinc-protoporphyrin.

Table II. Apoptosis index following I/R, as determined by terminal deoxynucleotidyl transferase dUTP nick end labeling.

\begin{tabular}{lc}
\hline Group & Apoptotic index (\%) \\
\hline Control & $7.92 \pm 2.31$ \\
I/R & $18.17 \pm 2.15^{\mathrm{a}}$ \\
I/R with CoPP pretreatment & $11.00 \pm 2.09^{\mathrm{b}}$ \\
I/R with CoPP and ZnPP pretreatment & $17.75 \pm 2.22$ \\
I/R with ZnPP pretreatment & $33.92 \pm 3.26^{\mathrm{b}}$ \\
\hline
\end{tabular}

Data are expressed as the mean \pm standard deviation. ${ }^{\mathrm{a}} \mathrm{P}<0.05$ vs. control; ${ }^{b} \mathrm{P}<0.05$ vs. I/R. I/R, ischemia-reperfusion; CoPP, cobalt-protoporphyrin; ZnPP, zinc-protoporphyrin.

lobules and inflammatory cell infiltration in portal duct areas was observed (Fig. 2B). CoPP pretreatment alleviated cellular swelling and the cellular morphology was not visibly different from the control group (Fig. 2C). The liver cells of the CoPP and $\mathrm{ZnPP}$ pretreatment group had an irregular arrangement and loss of the portal duct (Fig. 2D). Hydropic degeneration, an increase in cell size, irregular arrangement and a clear cytoplasmic shape of liver cells was observed in the ZnPP pretreatment group, and the liver sinusoid appeared narrow (Fig. 2E).

CoPP pretreatment promotes the expression of Bcl-2 following $I / R$. To investigate the association between the protein expression levels of HO-1 and cell death, HO-1 and Bcl-2 were detected by western blotting; $\beta$-actin served as an internal control. Compared with the control group, the protein expression levels of HO-1 were greater in the I/R group; this increase was inhibited by ZnPP pretreatment and further increased by CoPP pretreatment. The expression of $\mathrm{Bcl} 2$ followed a similar pattern (Fig. 3).

CoPP pretreatment inhibits apoptosis following $I / R$. To investigate the role of HO-1 in apoptosis a TUNEL assay was performed. In the CoPP pretreatment group, the percentage of apoptotic cells in the liver was reduced compared with the I/R group. The percentage of apoptotic cells in the CoPP and $\mathrm{ZnPP}$ pretreatment group was increased compared with the CoPP treatment group. The greatest percentage of apoptotic cells was observed in the ZnPP pretreatment group $(\mathrm{P}<0.05$; Table II and Fig. 4).

CoPP pretreatment inhibits caspase-3 expression following $I / R$. To assess the role of HO-1 in apoptosis, the expression of caspase- 3 was detected by immunohistochemistry. Caspase-3 expression is a marker of apoptotic cell death (14); therefore, apoptotic cells were identified by detecting caspase-3 expression by immunohistochemistry. Caspase-3 staining was greater in the I/R group compared with the control group $(\mathrm{P}<0.05)$; this increase was attenuated by CoPP pretreatment and enhanced by ZnPP pretreatment ( $\mathrm{P}<0.05$, Table III and Fig. 5). These results indicated that $\mathrm{HO}-1$ may prevent cells from damage and death by inhibiting the expression of caspase- 3 .

\section{Discussion}

The results of the present study demonstrated that CoPP pretreatment was associated with the attenuation of injury induced by hepatic I/R, whereas this injury was exacerbated by ZnPP pretreatment. Results of western blotting and TUNEL assays indicated that the CoPP pretreatment group had greater protein expression levels of $\mathrm{HO}-1$ and $\mathrm{Bcl}-2$, and a reduced percentage of apoptotic cells. In addition, liver cell injury was increased following ZnPP pretreatment. Furthermore, the protein expression levels of HO-1 were decreased following pretreatment with ZnPP. The results of the present study suggested that preconditioning with CoPP may have a protective effect and attenuate I/R injury in liver transplantation through induction of HO-1 expression. Preconditioning with ZnPP downregulated HO-1 expression and aggravated cell injury. Therefore, HO-1 expression may serve an important role in the protection of cells against I/R injury.

Previous studies have identified that mice lacking HO-1 $\left(\mathrm{Hmox}^{-} /\right)$are more susceptible to I/R injury compared with $\mathrm{Hmox}^{-/+}$and $\mathrm{Hmox}^{+/+}$animals, indicating that HO-1 may serve a protective role in I/R injury $(3,15,16)$. A further study revealed that HO-1 was the most sensitive index of stress, and 

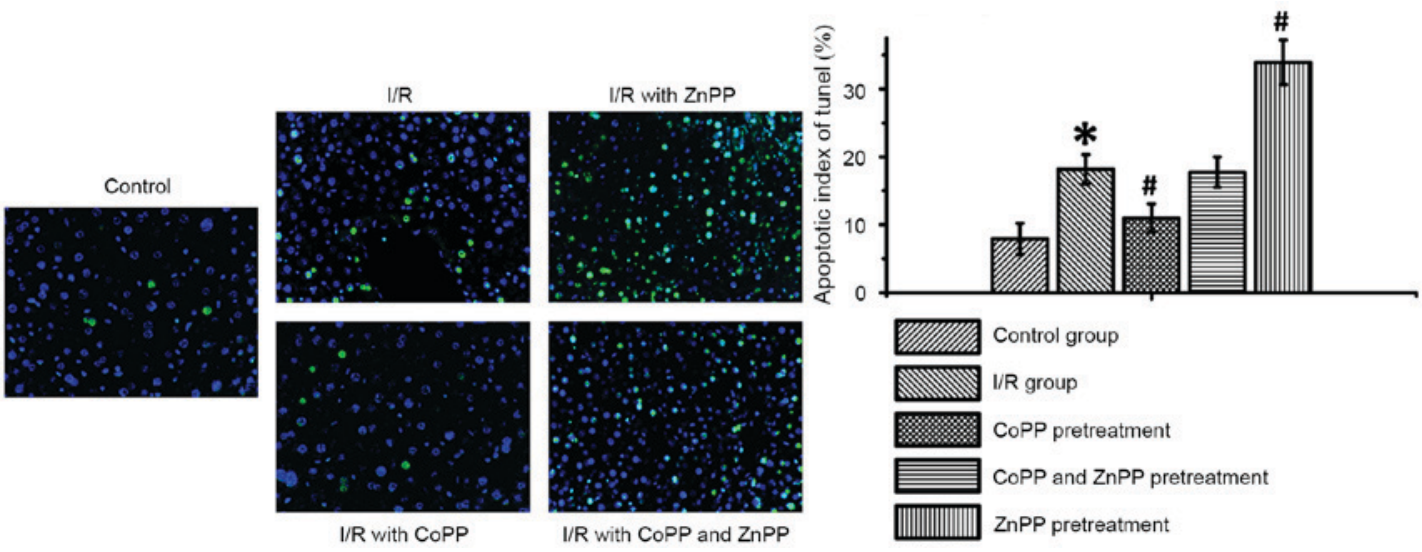

Figure 4. Apoptosis following I/R injury. Liver tissues were obtained from mice following liver I/R injury, in the absence or presence of pretreatment with CoPP and/or ZnPP, and apoptosis was detected using terminal deoxynucleotidyl transferase dUTP nick end labeling. I/R injury increased apoptosis; this increase was attenuated by CoPP pretreatment and enhanced by ZnPP pretreatment. Data are expressed as the mean \pm standard deviation. ${ }^{*} \mathrm{P}<0.05$ vs. control; " $\mathrm{P}<0.05$ vs I/R. Original magnification, x400. I/R, ischemia-reperfusion; CoPP, cobalt-protoporphyrin; ZnPP, zinc-protoporphyrin; Apoptotic index of tunel=Apoptotic cell number/total cell number, apoptotic cell showed as green fluorescence, and the blue fluorescence represents counterstaining with DAPI.

I/R

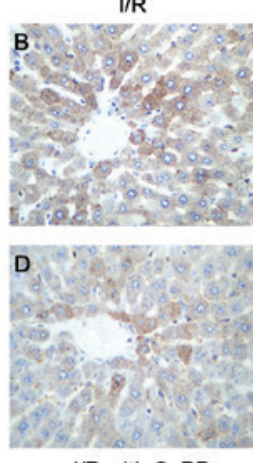

I/R with CoPP
I/R with ZnPP

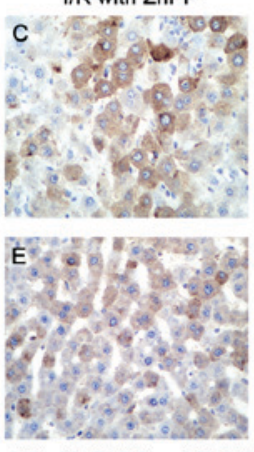

I/R with CoPP and ZnPP
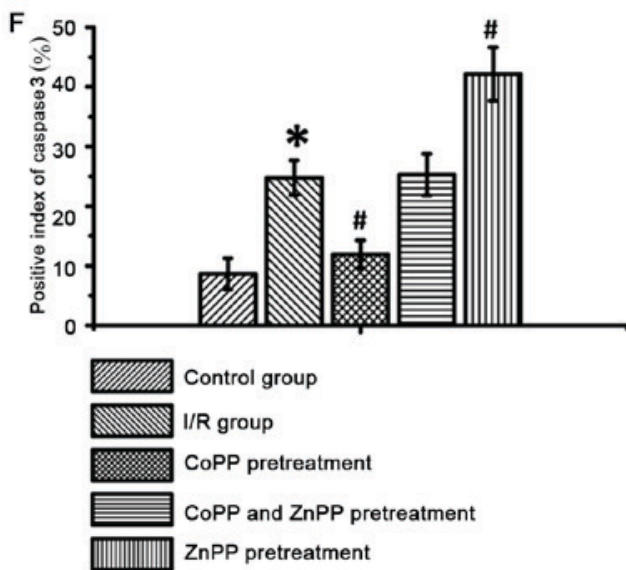

Figure 5. Caspase-3 expression following I/R injury. Liver tissues were obtained from mice following liver I/R injury, in the absence or presence of pretreatment with CoPP and/or ZnPP, and caspase-3 expression was detected by immunohistochemistry. Brown indicates positive staining. (A) Control, (B) I/R, (C) I/R with ZnPP pretreatment, (D) I/R with CoPP pretreatment and (E) I/R with CoPP and ZnPP pretreatment. (F) Quantification of caspase-3 staining demonstrated that caspase-3 expression was increased by I/R injury; this increase was reduced by CoPP pretreatment and enhanced by $\mathrm{ZnPP}$ pretreatment. Data are expressed as the mean \pm standard deviation. ${ }^{*} \mathrm{P}<0.05$ vs. control; ${ }^{*} \mathrm{P}<0.05$ vs. I/R. Original magnification, $\mathrm{x} 400$. I/R, ischemia-reperfusion; CoPP, cobalt-protoporphyrin; ZnPP, zinc-protoporphyrin.

Table III. Caspase-3 expression following I/R, as detected by immunohistochemistry.

\begin{tabular}{lc}
\hline Group & Positive index $(\%)$ \\
\hline Control & $8.67 \pm 2.61$ \\
I/R & $24.75 \pm 2.93^{\mathrm{a}}$ \\
I/R with CoPP pretreatment & $11.92 \pm 2.35^{\mathrm{b}}$ \\
I/R with CoPP and ZnPP pretreatment & $25.25 \pm 3.50$ \\
I/R with ZnPP pretreatment & $42.17 \pm 4.47^{\mathrm{b}}$
\end{tabular}

Data are expressed as the mean \pm standard deviation. ${ }^{\mathrm{a}} \mathrm{P}<0.05$ vs. control; ${ }^{b} \mathrm{P}<0.05$ vs. I/R. I/R, ischemia-reperfusion; CoPP, cobalt-protoporphyrin; ZnPP, zinc-protoporphyrin.

knockdown of HO-1 expression by small interfering (si)RNA may promote cell death (17).
Apoptosis serves an important role in hepatic I/R injury; the underlying mechanisms may involve death receptors including Fas and tumor necrosis factor $\alpha$, and/or mitochondrial dysfunction induced by cellular stress (1). The pro-apoptotic Bcl-2 and caspase families are the primary signaling pathways. The metabolic products of heme degraded by $\mathrm{HO}-1$, ferrous iron, biliverdin and carbon monoxide, are considered vital to protect cells from inflammation, apoptosis and oxidative stress $(18,19)$. Ferrous iron released by HO- 1 may induce expression of the $\mathrm{Fe}^{2+}$ sequestering protein ferritin, which may inhibit apoptosis to protect endothelial and liver cells (20). In addition, carbon monoxide may significantly decrease mRNA expression levels of the pro-apoptotic $\mathrm{Bcl}-2$-associated $\mathrm{X}$ protein and promote the expression of Bcl-2 $(21,22)$.

The induction of the caspase cascade results in cell death. Caspase-3 is the 'effector' protease of the apoptosis cascade and serves an important role in programmed cell death (23). Caspase-3 knockdown by siRNA decreased cell damage 
and improved cell survival (24). In the present study, CoPP pretreatment decreased caspase- 3 expression and the degree of cell death compared with I/R alone. Therefore, high expression levels of HO-1 induced by CoPP may inhibit caspase-3 expression and promote $\mathrm{Bcl}-2$ expression to protect cells from I/R-induced injury and apoptosis.

Liver transplantation is an important treatment for patients with hepatocellular carcinoma (25). I/R injury is an inevitable consequence of organ transplantation and limits long-term survival (26). I/R injury was described by Jennings et al (27) in 1960 as damage that occurred when blood supply returned to tissue following a period of ischemia. The findings of the present study suggested that apoptosis is important in I/R injury, and that CoPP may protect liver cells by upregulating HO-1 expression. CoPP may therefore be a potential therapeutic agent for the treatment of ischemic diseases.

\section{References}

1. Ben-Ari Z, Issan Y, Katz Y, Sultan M, Safran M, Michal LS Nader GA, Kornowski R, Grief F, Pappo O and Hochhauser E: Induction of heme oxygenase-1 protects mouse liver from apoptotic ischemia/reperfusion injury. Apoptosis 18: 547-555, 2013.

2. Maines MD: The heme oxygenase system: A regulator of second messenger gases. Annu Rev Pharmacol Toxicol 37: 517-554, 1997.

3. Wang CF, Wang ZY and Li JY: Dual protective role of HO-1 in transplanted liver grafts: A review of experimental and clinical studies. World J Gastroenterol 17: 3101-3108, 2011.

4. Liu B and Qian JM: Cytoprotective role of heme oxygenase-1 in liver ischemia reperfusion injury. Int J Clin Exp Med 8: 19867-19873, 2015.

5. Lai IR, Ma MC, Chen CF and Chang KJ: The protective role of heme oxygenase-1 on the liver after hypoxic preconditioning in rats. Transplantation 77: 1004-1008, 2004.

6. Yun N, Eum HA and Lee SM: Protective role of heme oxygenase-1 against liver damage caused by hepatic ischemia and reperfusion in rats. Antioxid Redox Signal 13: 1503-1512, 2010.

7. Lin HY, Tsai CH, Lin C, Yeh WL, Tsai CF, Chang PC, Wu LH and Lu DY: Cobalt protoporphyrin upregulates cyclooxygenase-2 expression through a heme oxygenase-independent mechanism. Mol Neurobiol 53: 4497-4508, 2016.

8. Huang HF, Zeng Z, Wang KH, Zhang HY, Wang S, Zhou WX, Wang ZB, Xu WG and Duan J: Heme oxygenase-1 protects rat liver against warm ischemia/reperfusion injury via TLR2/TLR4-triggered signaling pathways. World J Gastroenterol 21: 2937-2948, 2015.

9. Chi X, Yao W, Xia H, Jin Y, Li X, Cai J and Hei Z: Elevation of HO-1 expression mitigates intestinal ischemia-reperfusion injury and restores tight junction function in a rat liver transplantation model. Oxid Med Cell Longev 2015: 986075, 2015.

10. Guide for the Care and Use of Laboratory Animals. The National Academies Press, Washington, DC, 2011

11. Selzner N, Selzner M, Jochum W and Clavien PA: Ischemic preconditioning protects the steatotic mouse liver against reperfusion injury: An ATP dependent mechanism. J Hepatol 39: 55-61, 2003.

12. Gavrieli Y, Sherman Y and Ben-Sasson SA: Identification of programmed cell death in situ via specific labeling of nuclear DNA fragmentation. J Cell Biol 119: 493-501, 1992.
13. Liu G, Wang T, Wang T, Song $\mathrm{J}$ and Zhou Z: Effects of apoptosis-related proteins caspase-3, Bax and Bcl-2 on cerebral ischemia rats. Biomed Rep 1: 861-867, 2013.

14. Eisenhardt SU, Weiss JB, Smolka C, Maxeiner J, Pankratz F, Bemtgen X, Kustermann M, Thiele JR, Schmidt Y, Bjoern Stark G, et al: MicroRNA-155 aggravates ischemia-reperfusion injury by modulation of inflammatory cell recruitment and the respiratory oxidative burst. Basic Res Cardiol 110: 32, 2015.

15. Fortes GB, Alves LS, de Oliveira R, Dutra FF, Rodrigues D, Fernandez PL, Souto-Padron T, De Rosa MJ, Kelliher M, Golenbock D, et al: Heme induces programmed necrosis on macrophages through autocrine TNF and ROS production. Blood 119: 2368-2375, 2012.

16. Devey L, Ferenbach D, Mohr E, Sangster K, Bellamy CO, Hughes $\mathbf{J}$ and Wigmore SJ: Tissue-resident macrophages protect the liver from ischemia reperfusion injury via a heme oxygenase-1-dependent mechanism. Mol Ther 17: 65-72, 2009.

17. Yang Y, Wang J, Li Y, Fan C, Jiang S, Zhao L, Di S, Xin Z, Wang B, Wu G, et al: $\mathrm{HO}-1$ signaling activation by pterostilbene treatment attenuates mitochondrial oxidative damage induced by cerebral ischemia reperfusion injury. Mol Neurobiol 53: 2339-2353, 2016.

18. Stocker R, Yamamoto Y, McDonagh AF, Glazer AN and Ames BN: Bilirubin is an antioxidant of possible physiological importance. Science 235: 1043-1046, 1987.

19. Harder Y, Amon M, Schramm R, Rücker M, Scheuer C, Pittet B, Erni D and Menger MD: Ischemia-induced up-regulation of heme oxygenase-1 protects from apoptotic cell death and tissue necrosis. J Surg Res 150: 293-303, 2008.

20. Berberat PO, Katori M, Kaczmarek E, Anselmo D, Lassman C, Ke B, Shen X, Busuttil RW, Yamashita K, Csizmadia E, et al: Heavy chain ferritin acts as an antiapoptotic gene that protects livers from ischemia-reperfusion injury. FASEB J 17: 1724-1726, 2003.

21. Han HH, Lim YM, Park SW, Lee SJ, Rhie JW and Lee JH: Improved skin flap survival in venous ischemia-reperfusion injury with the use of adipose-derived stem cells. Microsurgery 35: 645-652, 2015.

22. Nakao A, Kimizuka K, Stolz DB, Neto JS, Kaizu T, Choi AM, Uchiyama T, Zuckerbraun BS, Nalesnik MA, Otterbein LE and Murase N: Carbon monoxide inhalation protects rat intestinal grafts from ischemia/reperfusion injury. Am J Pathol 163: $1587-1598,2003$.

23. Contreras JL, Vilatoba M, Eckstein C, Bilbao G, Anthony Thompson J and Eckhoff DE: Caspase- 8 and caspase- 3 small interfering RNA decreases ischemia/reperfusion injury to the liver in mice. Surgery 136: 390-400, 2004.

24. Hua P, Liu LB, Liu JL, Wang M, Jiang HQ, Zeng K, Yang YQ and Yang SR: Inhibition of apoptosis by knockdown of caspase-3 with siRNA in rat bone marrow mesenchymal stem cells. Exp Biol Med (Maywood) 238: 991-998, 2013.

25. Cotterell AH and Fisher RA: Ischemia/reperfusion injury and hepatocellular carcinoma recurrence after liver transplantation: Cancer at WIT's end? Dig Dis Sci 60: 2579-2580, 2015.

26. Dare AJ, Logan A, Prime TA, Rogatti S, Goddard M, Bolton EM, Bradley JA, Pettigrew GJ, Murphy MP and Saeb-Parsy K: The mitochondria-targeted anti-oxidant MitoQ decreases ischemia-reperfusion injury in a murine syngeneic heart transplant model. J Heart Lung Transplant 34: 1471-1480, 2015.

27. Jennings RB, Sommers HM, Smyth GA, Flack HA and Linn H: Myocardial necrosis induced by temporary occlusion of a coronary artery in the dog. Arch Pathol 70: 68-78, 1960. 